\section{Suprapubischer Katheter von Vorteil}

\author{
Die suprapubische Harnableitung ist für Patienten nach einer radikalen \\ Prostatektomie mit signifikant geringeren Schmerzen verbunden als ein \\ transurethraler Dauerkatheter.
}

$D^{\prime}$ e Vorteile einer suprapubischen Harnableitung, die postoperativen Schmerzen betreffend, müssen nicht mit einer höheren Strikturrate erkauft werden, wenn auf eine Schienung der vesikourethralen Anastomose verzichtet wird. Das haben Urologen um Nina Harke in einer Studie gezeigt.

Die 160 Studienteilnehmer, Männer mit klinisch auf das Organ begrenztem Prostatakrebs, wurden roboterunterstützt radikal prostatektomiert. Allen wurde intraoperativ ein transurethraler Katheter gelegt. In randomisierter 1:1-Zuordnung erhielt eine Gruppe der Männer nach Kontrolle der Anastomose auf Dichtigkeit zusätzlich einen suprapubischen Katheter; bei ihnen wurde der transurethrale Katheter am Morgen des ersten postoperativen Tages gezogen. In der anderen Gruppe blieb es bei der transurethralen Harnableitung.

Die Schmerzstärke wurde vom ersten postoperativen Tag bis zum Ziehen des Katheters am Tag fünf und auch noch am sechsten postoperativen Tag gemessen. Mit Ausnahme des ersten und letzten Tages war der Schmerzwert auf einer numerischen Skala von 0 bis 10 (schlimmste Schmerzen) in der Gruppe mit suprapubischem Katheter immer signifikant - absolut betrachtet um gut 1 Punkt - niedriger als in der Gruppe mit transurethralem Katheter. Die erzielten Werte sanken dabei im Lauf der Tage von 3-4 Punkten auf 1 Punkt. Trotz fehlender Schienung waren während der medianen Nachbeobachtungszeit von 22 Monaten keine funktionellen Nachteile nach suprapubischer Harnablei- tung im Vergleich zur Gruppe mit transurethraler Katheterisierung zu verzeichnen. Die Häufigkeit von Bakteriurien differierte ebenfalls nicht.

Einschränkend ist zu vermerken, dass insgesamt 29 Patienten - 23 in der Gruppe mit suprapubischem Katheter, sechs in der Kontrollgruppe - aus der Wertung fielen. Hauptgründe für den Verzicht auf die suprapubische Drainage waren intraoperative Komplikationen (14 Fälle), etwa die Notwendigkeit, den Blasenhals zu rekonstruieren (sieben Fälle); vier Patienten benötigten eine zusätzliche Hernienkorrektur.

Fazit: Harke und ihre Mitarbeiter schlagen vor zu diskutieren, ob das Legen eines suprapubischen Katheters in den Therapiestandard der roboterassistierten radikalen Prostatektomie aufzunehmen sei.

Dr. Robert Bublak

Harke $\mathrm{N}$ et al. Postoperative patient comfort in suprapubic drainage versus transurethral catheterization following robot-assisted radical prostatectomy: a prospective randomized clinical trial. World J Urol. 2017; 35: 389-94.

\title{
Jeder zweite Nierenstein vermeidbar
}

\section{Einer Kohortenstudie zufolge könnten fünf Lebensstilfaktoren für mehr als die Hälfte aller Nierenkonkremente verantwortlich sein.}

D ass Lebensstilfaktoren wie Gewicht, Flüssigkeitsaufnahme oder Kalziumzufuhr einen Einfluss auf das Risiko für Nierensteine haben, ist bekannt. Ärzte der Universität Rom und der Harvard Medical School haben nun untersucht, wie hoch ihr Beitrag zum erstmaligen Auftreten von symptomatischen Nierensteinen auf Bevölkerungsebene einzuschätzen ist. „Den BMI im Normbereich $\mathrm{zu}$ halten, ausreichend $\mathrm{zu}$ trinken, viel Obst und Gemüse und fettarme Milchprodukte zu essen (DASH-Diät), auf eine adäquate Kalziumzufuhr zu achten und wenig gesüßte Getränke zu trinken war mit einem klinisch relevant niedrigeren Risiko für Nierensteine assoziiert", schreiben die Autoren um Pietro M. Ferraro.

Für die Analyse wurden Daten von drei prospektiven Kohortenstudien Health Professionals Follow-up Study
(HPFS) und Nurses' Health Study (NHS) I und II - mit über 190.000 Männern und Frauen ausgewertet. Während der Beobachtungszeit von median elf Jahren wurde bei 6.449 Teilnehmern erstmals ein Nierenstein diagnostiziert. In jeder Kohorte waren alle fünf untersuchten Risikofaktoren unabhängig mit dem Steinrisiko assoziiert: Personen mit Übergewicht, weniger als zwei Liter Flüssigkeitsaufnahme pro Tag, einer deutlich von der DASH-Diät abweichenden Ernährung, einer hohen Kalziumzufuhr (im obersten Quintil) und mehr als vier gesüßten Getränken pro Woche hatten ein signifikant erhöhtes Risiko. Unter der Annahme, dass es sich bei diesen Assoziationen um kausale Zusammenhänge handelt, haben die Studienautoren die „population attributable fraction" (PAF) errechnet, also zu welchem Anteil die in den Kohor- ten aufgetretenen Nierensteine der Exposition zu einem bestimmten Risikofaktor zuzuschreiben waren. Den höchsten PAF-Wert erzielte in der HPFS-Kohorte eine geringe Flüssigkeitszufuhr; sie war demnach für 26,0 \% aller Fälle verantwortlich. In den NHS-Kohorten dagegen wurden die meisten Fälle, 21,8 \% und $18,9 \%$, auf einen erhöhten BMI zurückgeführt. Durch die fünf Risikofaktoren zusammen ließen sich je nach Kohorte 55,1-57,0 \% aller Steinkomplikationen erklären.

Fazit: Durch die vollständige Meidung der beschriebenen Risikofaktoren wäre gut die Hälfte aller beobachteten Fälle zu verhindern gewesen. Diese Größenordnung passt laut Ferraro und Kollegen zu den publizierten Abschätzungen bezüglich des Anteils von Umwelt und Genen an der Entstehung von Nierensteinen.

Dr. Beate Schumacher

Ferraro PM et al. Dietary and Lifestyle Risk Factors Associated with Incident Kidney Stones in Men and Women. J Urol. 2017; doi: 10.1016/j. juro.2017.03.124. 